\title{
Distinguishing esophageal from endotracheal intubation by measuring endotracheal tube cuff pressure
}

\author{
Brian J. Goentzel, Eric Larsen, Nicholas Shelburne, Darius Tolbert, William C. Culp Jr* and William E. Johnston \\ *Correspondence: wculp@sw.org \\ Department of Anesthesiology, Texas A\&M Health Science Center College of Medicine/Scott and White Healthcare, \\ Temple, Texas, USA.
}

\begin{abstract}
Background: It is unknown whether measuring endotracheal tube cuff pressure can accurately confirm tracheal intubation. Animal and human cadaveric studies show conflicting results. In anesthetized patients, we tested the hypothesis that the pressurevolume characteristics of the cuff located in the trachea would differ from that measured in the esophagus potentially making cuff pressure a useful tool to distinguish esophageal from tracheal intubation.

Methods: Thirty-four female and 35 male patients undergoing general anesthesia were intubated under direct laryngoscopy with $7.0 \mathrm{~mm}$ and $8.0 \mathrm{~mm}$ cuffed tubes, respectively, first in the esophagus and next in the trachea. Cuff pressures at each site were recorded during sequential one $\mathrm{mL}$ inflation volumes up to $10 \mathrm{~mL}$ or when cuff pressure exceeded $250 \mathrm{mmHg}$ and compared using the Wilcoxon signed rank test.

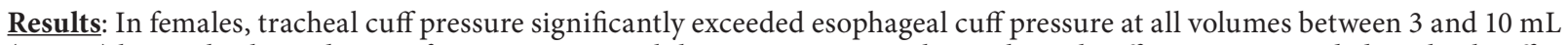
$(\mathrm{p}<0.05)$ but with a large degree of interpatient variability. In contrast, male esophageal cuff pressure exceeded tracheal cuff pressure at volumes less than $3 \mathrm{~mL}$ and did not differ between the two sites at higher cuff volumes.

Conclusions: In female patients, greater cuff pressures in the tracheal than esophageal location are attributed to the influence of less compliant cartilaginous rings. These findings were not replicated in male patients. However, the large degree of variability precludes the clinical application of this technique for accurate identification of cuff location in the trachea.
\end{abstract}

Keywords: Esophageal pressure, endotracheal intubation, cuff pressure, airway management

\section{Introduction}

Several outcome studies have clearly indicated that unrecognized esophageal intubation is a leading cause of brain damage and death in anesthetic practice [1-3]. Of particular concern are intubations performed outside the operating room, which have been fraught with an unacceptably high incidence of unidentified esophageal placement [4-8]. One study estimates the frequency of esophageal intubation in emergency airway management of critically ill patients to occur at a rate of $8 \%$ [9]. With the advent of pulse oximetry and capnography, undetected esophageal intubation has likely decreased, but remains a potentially catastrophic complication [10]. While multiple methods have been developed to delineate esophageal from tracheal intubation, virtually all modalities can fail [11-14]. Factors contributing to unrecognized esophageal intubation include unfavorable intubating conditions, absence of appropriate monitoring, and inexperienced personnel. Current methods of confirming proper endotracheal tube placement such as capnography and colorimetric carbon dioxide detection, though very successful and classified as "almost failsafe methods," [10] remain imperfect and have not completely resolved this critical issue.

Inherent structural differences exist between the esophagus and trachea that may allow differentiation of tube location by measuring cuff pressure. The esophagus is embedded in connective tissue known as the tunica adventitia, along with varying amounts of skeletal and smooth muscle. In addition, the lumen of the esophagus is normally closed. In contrast, the trachea is composed of 15-20 incomplete rigid C-shaped cartilaginous rings, which aid in protection and maintenance of airway patency. Theoretically, inflation of a cuffed tube in the trachea should produce low pressures at small cuff volumes with a marked nonlinear increase in cuff pressure once the confines of the tracheal wall have been met. Measuring the pressure exerted within the cuff of an endotracheal tube could be a novel and accurate method of confirming proper tube placement.

Recent studies performed in porcine models (anesthetized, non-paralyzed pigs [15] and ex vivo porcine tracheal-esophageal tissue preparations [16]) explored this method of confirming endotracheal tube placement. Both studies found that a curvilinear relationship existed between cuff volume and pressure in the tracheal location with an exponential increase in pressure at high cuff volumes. Unexpectedly, cuff pressure in the esophageal location exceeded that in the tracheal location at all cuff volumes which may be model dependent. 
In human cadavers, conflicting results were found where intracuff pressures did not differ between the two locations with cuff volumes exceeding $3 \mathrm{~mL}$ [17]. To date, no study has been performed in live human subjects to address this potentially important clinical issue.

We postulate that due to the inherent structural differences between the esophagus and trachea, measuring cuff pressure could provide a reliable method of confirming proper tube placement within the trachea. We therefore tested the hypothesis that intracuff pressure should initially be greater in the esophagus than the trachea at low $(<4 \mathrm{~mL})$ cuff volumes due to the closed lumen of the esophagus coupled with the fibromuscular structure of the esophageal wall lacking intrinsic support. Conversely, at higher intracuff volumes $(>4 \mathrm{~mL}$ ), the tracheal location should demonstrate markedly higher cuff pressure than the esophagus secondary to the less compliant cartilaginous rings. Secondarily, since tracheal volume has been reported to be linearly related to patient height [18], we anticipate that greater cuff volumes will be required to generate a pressure difference between tracheal and esophageal locations in taller patients.

\section{Methods}

The research study was approved by our institutional review board and written informed consent was obtained from each subject prior to study enrollment. Seventy adult subjects having elective surgery requiring endotracheal intubation were enrolled. Inclusion criteria included subjects greater than 18 years of age, predicted easy intubation, no risk of aspiration, and fasting longer than eight hours prior to surgery. Exclusion criteria included subject refusal, emergency surgery, increased risk of aspiration, known or expected difficult intubation, contraindication to the use of succinylcholine, and a history of esophageal or tracheal pathology. The study protocol was formulated by combining key aspects of the previous studies performed on porcine models $[15,16]$ and human cadaveric subjects [17], as well as integrating standard patient intubation techniques and monitoring to ensure amnesia, analgesia, and muscular relaxation during our study.

Prior to each study, two sterile cuffed tubes (Sheridan HVT endotracheal tube, Teleflex Medical Research Park, North Carolina; 7.0 mm (\#5-10314) for females and 8.0 mm (\#5-10316) for males) were prepared - one for esophageal intubation and the other for tracheal intubation. These tube sizes are standard for patients at our institution. Each tube's cuff was inflated ex vivo using a $10 \mathrm{~mL}$ syringe attached by a three-way stopcock to a calibrated, automated pressure manometer (SPER Scientific, model 840080 , Scottsdale, AZ) and to the pilot bulb on the cuffed tube. Initially each cuff was inflated to $10 \mathrm{~mL}$ to loosen cuff adherence from the plastic tube and to assess for leaks or deformities. Next, all air was removed, the manometer was re-zeroed, and the cuff was allowed to equilibrate with atmospheric pressure. Serial 1-mL incremental inflations of the cuff up to a total of $10 \mathrm{~mL}$ were made and baseline measurements were recorded. Next, the cuff was deflated and again allowed to equilibrate to atmospheric pressure.

Upon arrival in the operating room, standard monitors were applied to the patient, along with a bispectral index monitor (BIS Brain Monitor, Covidien, Mansfield, MA, USA) and a neurostimulator monitor over the ulnar nerve connected to an accelerometer attached to the subject's thumb (Draeger Infinity NMT, Lubeck, Germany). Each subject was preoxygenated with $100 \%$ oxygen for at least 3 minutes and received intravenous premedication using 1-2 mg midazolam, $50-100 \mathrm{mcg}$ fentanyl, and 60-100 mg lidocaine. Anesthesia was induced with $2-2.5 \mathrm{mg} / \mathrm{kg}$ of propofol and after assuring that mask ventilation was possible, muscular relaxation was provided with succinylcholine $(1.5 \mathrm{mg} / \mathrm{kg})$. Following fasciculations and greater than $98 \%$ decline in neuromuscular function, the study was begun.

Under direct laryngoscopy, the esophagus was intubated to a depth of $22 \mathrm{~cm}$ in females and $24 \mathrm{~cm}$ in males using the patient's lips as the anatomic reference point. The manometer/ stopcock/10 cc syringe set-up was attached to the pilot bulb of the cuff, and the manometer was zeroed. Serial esophageal cuff pressure measurements were performed and recorded at $1 \mathrm{~mL}$ increments up to $10 \mathrm{~mL}$ or until the cuff pressure reached $250 \mathrm{mmHg}$. The esophageal cuff was then deflated and the tube was removed from the esophagus and discarded. Again, using direct laryngoscopy, the other previously calibrated cuffed tube was placed into the trachea and advanced to the gender specific depth $(22 \mathrm{~cm}$ in females and $24 \mathrm{~cm}$ in males measured at the lips). Immediately following intubation and prior to any manometry measurements, the anesthesia circuit was attached to the cuffed tube and several breaths of $100 \%$ oxygen were provided to prevent hypoxia during the subsequent data collection period. Using the same manometer/stopcock/10 cc syringe set-up attached to the pilot bulb, the manometer was re-zeroed and cuff pressure measurements were repeated at $1 \mathrm{~mL}$ increments up to 10 $\mathrm{mL}$ or $250 \mathrm{mmHg}$ maximal pressure. Upon completion of data collection, the tracheal tube cuff was deflated to a pressure of $30 \mathrm{mmHg}$. Note that only one cuffed tube was in vivo at any time, therefore one tube exerted no pressure on the other. Bispectral index monitor readings were measured to ensure adequate depth of anesthesia during both intubations and throughout data collection. Additional doses of propofol (up to $1 \mathrm{mg} / \mathrm{kg}$ ) were administered to treat signs of inadequate anesthesia (tachycardia, hypertension, bispectral index value elevation $>40$ ) as necessary. Complete muscle paralysis throughout the study with train-of-four suppression was confirmed using the digital accelerometer. At any time, the attending anesthesiologist could stop the study if necessary to control the airway.

Based on data from the porcine study [15], a power analysis was performed. At a 4-mL cuff volume, a difference in cuff pressure between esophageal and tracheal locations of $20 \pm 2$ 


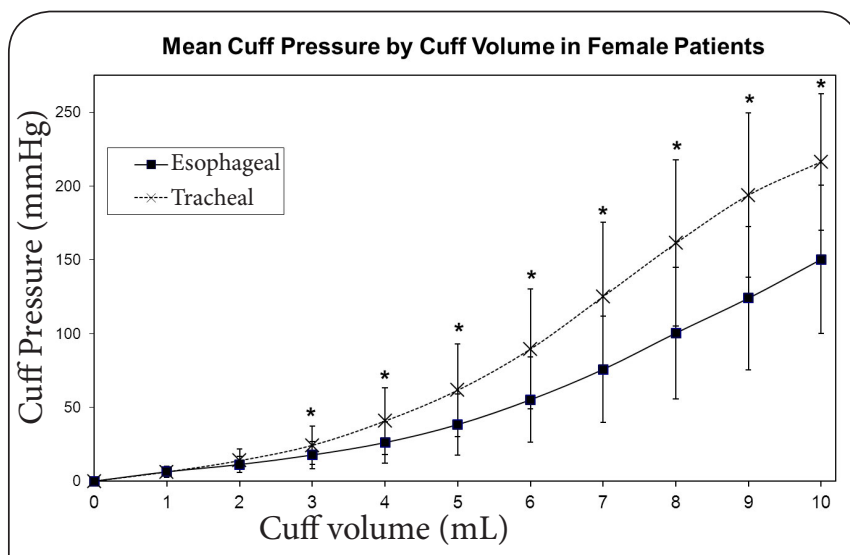

Figure 1. Intrinsic esophageal and tracheal cuff pressures in female patients during cuff inflation. With all cuff inflation volumes exceeding $2 \mathrm{~mL}$, tracheal cuff pressure significantly exceeded esophageal cuff pressure which demonstrates the potential value of measuring cuff pressure to confirm tracheal location. Error bars indicate one standard deviation from the mean; ${ }^{*}$ indicates $\mathrm{p}<0.05$ compared to corresponding esophageal cuff value.

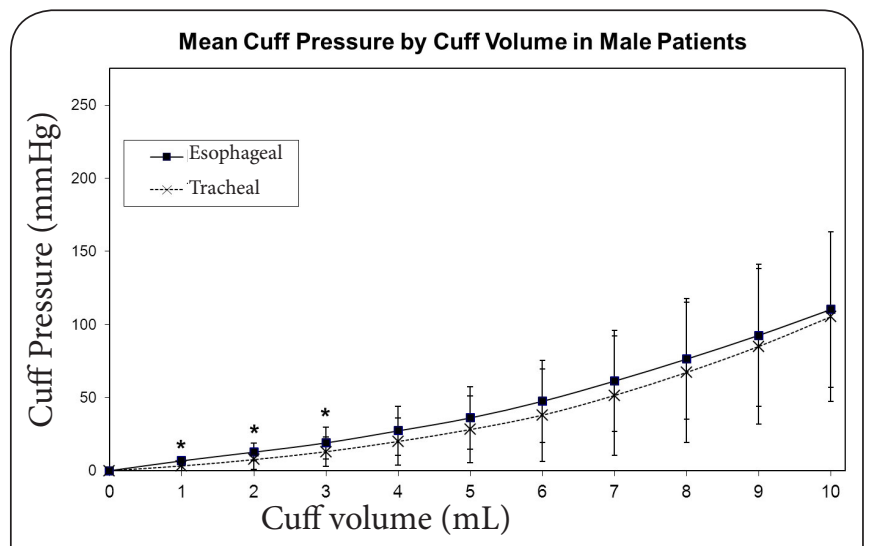

Figure 2. Intrinsic esophageal and tracheal cuff pressures in male patients during cuff inflation. With all cuff volumes greater than $3 \mathrm{~mL}$, there was no significant difference in cuff pressures measured in tracheal or esophageal locations. Error bars indicate one standard deviation from the mean; *indicates $\mathrm{p}<0.05$ compared to corresponding tracheal cuff value.

Table 1. Population statistics.

\begin{tabular}{lll}
\hline & Female & Male \\
\hline Number & 34 & 35 \\
Age & $50 \pm 18$ & $51 \pm 17$ \\
Height $(\mathrm{cm})$ & $162 \pm 7$ & $178 \pm 9$ \\
Weight $(\mathrm{kg})$ & $84 \pm 21$ & $93 \pm 25$ \\
\hline
\end{tabular}

Data are expressed as the mean \pm SD.

$\mathrm{mmHg}$ would require 24 patients in each group for $80 \%$ power at $p<0.05$. Consequently, the study was continued until 35
Table 2. Mean tracheal and esophageal cuff pressure differences by cuff volume.

\begin{tabular}{lll}
\hline $\begin{array}{l}\text { Cuff Volume } \\
(\mathbf{m L})\end{array}$ & $\begin{array}{l}\text { Cuff Pressure Difference } \\
\text { in Females }(\mathbf{m m H g})\end{array}$ & $\begin{array}{l}\text { Cuff Pressure Difference } \\
\text { in Males }(\mathbf{m m H g})\end{array}$ \\
\hline 1 & $-0.1 \pm 4.7(0.73)$ & $-3.5 \pm 4.5(0.0001)$ \\
2 & $2.6 \pm 9.0(0.16)$ & $-5.1 \pm 9.1(0.0024)$ \\
3 & $6.5 \pm 14.4(0.015)$ & $-6.0 \pm 15.0(0.026)$ \\
4 & $14.6 \pm 23.9(0.0011)$ & $-7.25 \pm 23.0(0.055)$ \\
5 & $23.2 \pm 32.3(0.0006)$ & $-7.9 \pm 29.9(0.096)$ \\
6 & $34.3 \pm 42.6(0.0005)$ & $-9.5 \pm 39.4(0.11)$ \\
7 & $49.3 \pm 51.6(<0.0001)$ & $-9.8 \pm 48.3(0.15)$ \\
8 & $60.9 \pm 58.0(<0.0001)$ & $-9.1 \pm 56.8(0.25)$ \\
9 & $69.9 \pm 63.7(<0.0001)$ & $-7.6 \pm 64.9(0.54)$ \\
10 & $66.1 \pm 59.9(<0.0001)$ & $-5.0 \pm 67.6(0.77)$ \\
\hline
\end{tabular}

Data are reported as mean \pm SD ( $p$ value). Difference is calculated as intrinsic tracheal cuff pressure-intrinsic esophageal cuff pressure.

patients were enrolled in each group. For statistical analysis, intrinsic cuff pressure was calculated by subtracting the baseline ex vivo cuff pressure from the measured cuff pressure for each cuff inflation volume in the different locations. Using the Wilcoxon signed rank test, tracheal and esophageal intrinsic pressures were compared at each cuff inflation volume for male and female patients with $p<0.05$ considered statistically significant (Analyse-It software ${ }^{\oplus}$, Leeds, UK). Data are expressed as mean \pm standard deviation. Linear regression and Pearson's correlation coefficient were determined for the cuff volume required for tracheal cuff pressure-esophageal cuff pressure to equal or exceed $20 \mathrm{mmHg}$ and patient height for male and female patients. Lastly, in ex vivo experiments, ten 7.0 $\mathrm{mm}$ and ten $8.0 \mathrm{~mm}$ cuffed tubes had their cuffs maximally inflated with $10 \mathrm{~mL}$ volume and the outside diameters were measured using digital calipers.

\section{Results}

Seventy subjects were enrolled in this study (34 female, 35 male) with one patient excluded due to technical difficulties with intubation. Esopheageal and endotracheal cuff pressure monitoring took approximately $40-60$ seconds each. No hypoxemia or other complications occurred. (Table 1) displays the population statistics for the remaining patients included in the data analysis.

Mean intrinsic esophageal and tracheal cuff pressures for females and males are represented graphically in (Figures $\mathbf{1}$ and $\mathbf{2}$ ). In females, initial cuff pressure at 1-2 mL cuff volumes was similar between esophageal and tracheal locations. Between 3 and $10 \mathrm{~mL}$ of inflation volume, tracheal cuff pressure be-came significantly higher than esophageal cuff pressure $(p<0.05)$. In contrast, in male patients, esophageal cuff pressure exceeded tracheal cuff pressure at initial cuff volumes up to $3 \mathrm{~mL}$ and then both increased similarly with greater cuff inflation. (Table 2) reports the mean, standard deviation, and $p$ values for the 


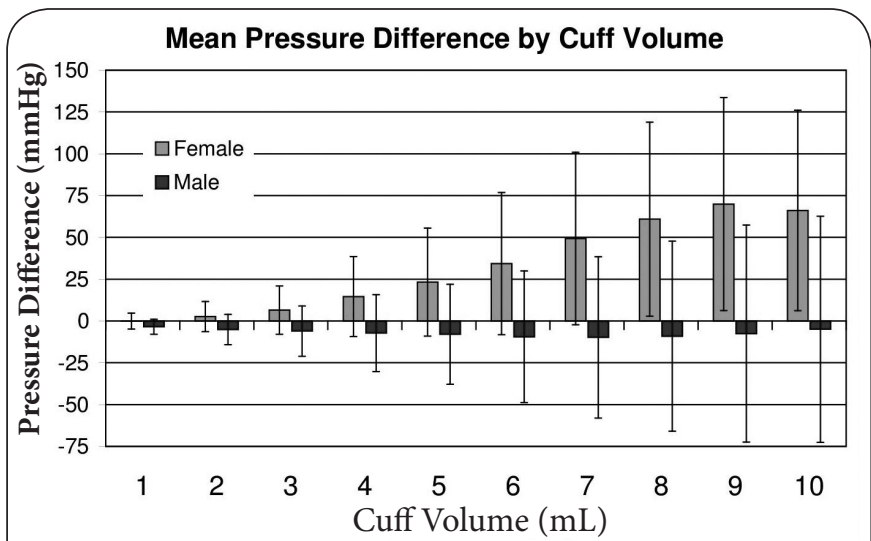

Figure 3. The bar graph shows the mean difference between tracheal and esophageal cuff pressures for male and female subjects during cuff inflation. Note the greater pressure difference in female patients in contrast to male patients. Error bars show one standard deviation from the mean.

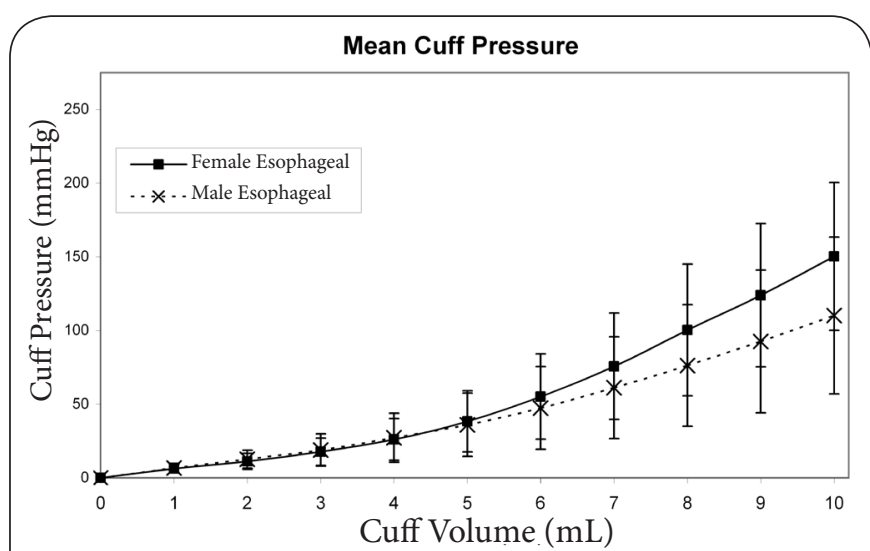

Figure 4. Mean esophageal cuff pressures in males and female patients during cuff inflation. Note a similar pressure response in both genders to cuff inflation. Error bars show one standard deviation from the mean.

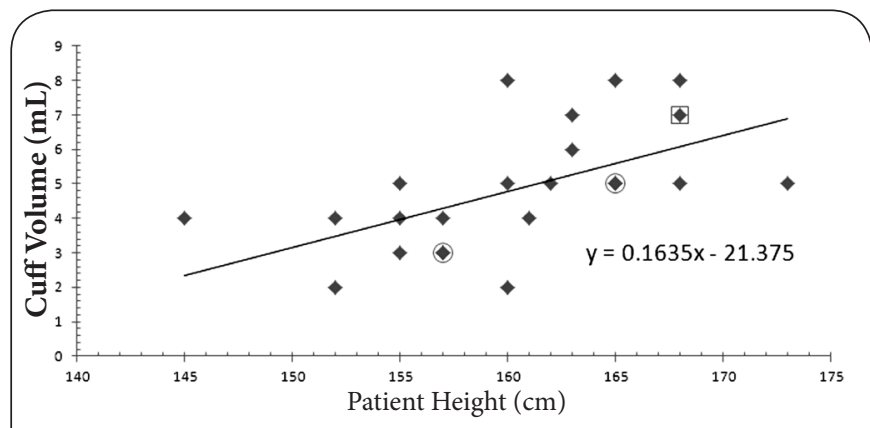

Figure 5. The cuff volume required for tracheal-esophageal cuff pressure gradient to equal or exceed $20 \mathrm{mmHg}$ is plotted against patient height for female patients. A significant positive linear correlation was found $(\mathrm{r}=0.58 ; \mathrm{p}<0.01)$ indicating that taller female patients have larger tracheas. A similar correlation was not found in male patients. (solid diamond indicates one patient; solid diamond with open square indicates two patients; solid diamond with open circle indicates 3 patients).
Table 3. Cuff diameters measured after 10-mL inflation volume in ten 8.0 and ten $7.0 \mathrm{~mm}$ standard endotracheal tubes.

\begin{tabular}{lcc}
\hline Tube number & $\mathbf{8 . 0 ~} \mathbf{~ m m}$ & $\mathbf{7 . 0 ~} \mathbf{~ m}$ \\
\hline 1 & 26.1 & 25.6 \\
2 & 27.6 & 24.8 \\
3 & 26.8 & 25.9 \\
4 & 26.8 & 26.2 \\
5 & 27.1 & 26.0 \\
6 & 28.3 & 26.0 \\
7 & 26.8 & 26.3 \\
8 & 26.6 & 25.9 \\
9 & 26.3 & 25.8 \\
10 & 27.0 & 25.8 \\
\hline \multicolumn{2}{l}{ Data are reported as measured in $\mathrm{mm}$}
\end{tabular}

differences between mean esophageal and tracheal cuff pressures at each cuff volume.

(Figure 3) displays the mean differences between esophageal and tracheal cuff pressure measurements at all cuff volumes. For females, the difference in cuff pressure increased as the volume inflated increased. This difference was greatest at $9 \mathrm{~mL}$ cuff volume, where mean tracheal cuff pressure was 70 $\mathrm{mmHg}$ higher (SD $64 \mathrm{mmHg}, \mathrm{p}<0.0001)$ than mean esophageal cuff pressure. In contrast, in male patients, mean esophageal pressure was similar to tracheal pressure at all data points above $3 \mathrm{~mL}$ cuff volume. As a comparison, (Figure 4) shows esophageal pressures for male and female patients where similar pressure responses were found with cuff inflation regardless of gender.

Twenty-six female patients but only 10 male patients had tracheal cuff pressure-esophageal cuff pressure equal to or exceeding $20 \mathrm{mmHg}$. A linear correlation was found in female patients when the cuff volume required to produce this pressure gradient was compared with patient height $(r=0.58 ; p<0.01$; Figure 5$)$. A greater cuff volume was required in taller female patients to generate a $20 \mathrm{mmHg}$ pressure gradient. Since esophageal cuff pressure exceeded tracheal cuff pressure in the majority of measurements in male patients, this relationship was only seen in 10 patients and a linear correlation between cuff volume and patient height could not be established $(r=0.24 ; p=0.48)$.

(Table 3) displays the outside diameters of the inflated 7.0 $\mathrm{mm}$ and $8.0 \mathrm{~mm}$ endotracheal tubes measured with a digital caliper. The $7.0 \mathrm{~mm}$ tube had a mean cuff diameter of $25.8 \pm 0.4$ $\mathrm{mm}$ and the $8.0 \mathrm{~mm}$ tube had a cuff diameter of $26.9 \pm 0.6 \mathrm{~mm}$.

\section{Discussion}

Confirmation of correct endotracheal tube placement is a critical step in airway management. Routinely, this is readily achieved with high success in the operating room by skilled 
anesthesiologists using capnography and pulse oximetry. However, in emergency situations outside of the operating room environment, such as in pre-hospital or third world environments, these tools may not be available. The selfinflating bulb and colorimetric carbon dioxide detector are inexpensive devices that aid in detecting inadvertent esophageal intubation, though each have limitations $[10,19]$. Of note, patients in cardiac arrest may not generate adequate end-tidal carbon dioxide. These and other limitations present an opportunity for a new technique to confirm correct endotracheal tube placement in the emergency situation.

This prospective study in live, human subjects was designed to determine whether measuring cuff pressure could detect esophageal location of a cuffed tube, resolving conflicting existing data. Our study did show a significantly greater cuff pressure in the tracheal location than esophageal location at cuff volumes exceeding $3 \mathrm{~mL}$ in females. This finding supports our hypothesis that, as the cuff expands in the tracheal location to reach the confines of the less compliant cartilaginous rings, further inflation causes a marked increase in cuff pressure. Potentially, measuring cuff pressure might be a useful tool to distinguish esophageal from tracheal intubation in female patients. In contrast, male subjects did not display this relationship where esophageal and tracheal cuff pressures were similar with cuff volumes exceeding $3 \mathrm{~mL}$ thus rejecting our hypothesis.

Using a porcine model, Hsu et al., demonstrated a very large difference in cuff pressures, with esophageal cuff pressure exceeding tracheal cuff pressure measured throughout cuff inflation [15]. However, the animals were placed in a dorsal recumbent position and were not paralyzed. The same laboratory repeated the study using tracheal-esophageal tissue preparations harvested from adult pigs with similar conclusions [16]. Using a human cadaveric model, Russo et al., found conflicting results with esophageal and tracheal pressures demonstrating no significant difference with cuff volumes exceeding $3 \mathrm{~mL}$ [17]. However, that study may be confounded by postmortem changes which could have altered tissue compliances.

In the present study, anesthetized males and female patients showed very different tracheal cuff pressure-volume relationships while the esophageal cuff pressure-volume curves for both genders appeared similar (Figure 4). We postulate that differences in cuff pressure between the tracheal location in males and females might be due to the use of a different size tube and the relative size of the fully inflated cuff in each gender's tracheal anatomy.

Kamel et al., reported that the maximum mean transverse diameter of the normal human trachea was $22.9 \pm 2.6 \mathrm{~mm}$ in females, and $27.1 \pm 3.4 \mathrm{~mm}$ in males [18]. If it is assumed that the trachea can be represented as a circle, the radiographic diameters would have a cross sectional area of $411.9 \mathrm{~mm}^{2}$ in females and $576.8 \mathrm{~mm}^{2}$ in males. Using the measured inflated cuff diameters (Table 3), a $7.0 \mathrm{~mm}$ tube would have a maximal cross sectional area of $523.7 \mathrm{~mm}^{2}$ and an $8.0 \mathrm{~mm}$ tube would have an area of $569.3 \mathrm{~mm}^{2}$. If the measured cuff cross sectional area is divided by the tracheal cross sectional area based on radiographic data for each gender, the $7.0 \mathrm{~mm}$ tube has a ratio of 1.27 in females. In comparison, the $8.0 \mathrm{~mm}$ tube has a ratio of 0.99 in the sagittal plane in males. These findings indicate a much greater probability that the $7.0 \mathrm{~mm}$ tube cuff would contact the tracheal wall during inflation in females earlier than the $8.0 \mathrm{~mm}$ tube cuff in males and may provide an explanation for the gender differences in tracheal cuff pressure-volume relations. Similar results are found using sagittal diameters of the tracheal air column measured on posteroanterior and lateral chest radiographs in patients [20]. Additionally, this proportional difference between tracheal and cuff areas might explain the higher incidence of postoperative hoarseness and sore throat reported by female compared to male patients [21-22].

\section{Limitations}

There are several potential limitations to our study. We only studied one particular brand of endotracheal tube and the cuff compliances of tubes made by other manufacturers may be different. Our results could have been affected by the use of succinylcholine, which might have changed the compliance of the esophagus by facilitating paralysis and muscle relaxation. Consequently, our results may differ from emergent intubations in non-paralyzed patients such as encountered outside the operating room. In addition, our study population consisted entirely of subjects undergoing elective surgery at our institution with a mean age of approximately 50 years. Inclusion of younger or healthier subjects in the data analysis may have different results, as the compliance and size of the esophagus and trachea can change with age, height, weight, and underlying disease process $[\mathbf{1 8 , 2 0}$. Similar to the study of tracheal morphology by Kamel [18] where a significant positive correlation between tracheal volume and patient height was found in female but not male patients, we found that greater cuff volumes as a patient's height increased to achieve a cuff pressure gradient above $20 \mathrm{mmHg}$ were necessary only in female patients.

Finally, our assumption that the lumen of the trachea is circular potentially overestimates actual cross sectional area but this assumption was made in both genders and only serves to illustrate that a relatively larger tube was used in female patients than in male patients. This potential discrepancy in tube size relative to tracheal lumen size may account for our findings. Although a statistically significant difference was found in female patients between esophageal and tracheal cuff pressures at higher cuff volumes $(>3 \mathrm{~mL})$, there still remains a high degree of variability among patients. The standard deviation of these differences is nearly as large as the mean value which limits the creation of a clinical standard that could apply to the general population. Any test to verify endotracheal intubation mandates a high level of accuracy 
since a false positive result could be life threatening. The measurement of cuff pressure does not meet this standard.

\section{Conclusion}

In conclusion, this study supports our initial hypothesis in female patients in whom tracheal cuff pressure increases rapidly and becomes significantly greater than esophageal pressure at cuff volumes $>3 \mathrm{~mL}$ but with a large degree of interpatient variability. In contrast, the data from male subjects do not support this hypothesis which we believe relates to the conventional use of an $8.0 \mathrm{~mm}$ cuffed tube relative to the reported larger size of the adult male trachea. This study in anesthetized humans clearly shows that the measurement of cuff pressure cannot accurately identify tracheal tube location and cannot be recommended for clinical use.

Competing interests

The authors declare that they have no competing interests.

Authors' contributions

\begin{tabular}{|l|c|c|c|c|c|c|}
\hline Authors' contributions & BJG & EL & NS & DT & WCC & WEJ \\
\hline Research concept and design & -- & $\checkmark$ & -- & -- & $\checkmark$ & $\checkmark$ \\
\hline Collection and/or assembly of data & $\checkmark$ & $\checkmark$ & $\checkmark$ & $\checkmark$ & $\checkmark$ & $\checkmark$ \\
\hline Data analysis and interpretation & $\checkmark$ & -- & -- & - & $\checkmark$ & $\checkmark$ \\
\hline Writing the article & $\checkmark$ & -- & -- & -- & -- & $\checkmark$ \\
\hline Critical revision of the article & -- & -- & -- & -- & $\checkmark$ & $\checkmark$ \\
\hline Final approval of article & $\checkmark$ & $\checkmark$ & $\checkmark$ & $\checkmark$ & $\checkmark$ & $\checkmark$ \\
\hline Statistical analysis & -- & -- & -- & -- & -- & $\checkmark$ \\
\hline
\end{tabular}

Acknowledgement

Funding in part from a Resident Mentor Award grant from Scott \& White Healthcare, along with routine departmental and institutional sources.

Publication history

EIC: Joseph Varon, University of Texas Medical Branch, USA. Received: 25-Sep-2013 Revised: 04-Nov-2013

Accepted: 14-Nov-2013 Published: 06-Dec-2013

\section{References}

1. Keenan RL and Boyan CP. Cardiac arrest due to anesthesia. A study of incidence and causes. JAMA. 1985; 253:2373-7. | Article | PubMed

2. Cheney FW, Posner KL, Lee LA, Caplan RA and Domino KB. Trends in anesthesia-related death and brain damage: A closed claims analysis. Anesthesiology. 2006; 105:1081-6. | Article | PubMed

3. Holland R, Webb RK and Runciman WB. The Australian Incident Monitoring Study. Oesophageal intubation: an analysis of 2000 incident reports. Anaesth Intensive Care. 1993; 21:608-10. | PubMed

4. Andersen $\mathrm{KH}$ and Schultz-Lebahn T. Oesophageal intubation can be undetected by auscultation of the chest. Acta Anaesthesiol Scand. 1994; 38:580-2. | Article | PubMed

5. Charters $P$. Normal chest expansion with oesophageal placement of a tracheal tube. Anaesthesia. 1989; 44:365. | Article | PubMed

6. Vaghadia $\mathrm{H}$, Jenkins $L C$ and Ford RW. Comparison of end-tidal carbon dioxide, oxygen saturation and clinical signs for the detection of oesophageal intubation. Can J Anaesth. 1989; 36:560-4. | Article | PubMed

7. Kelly JJ, Eynon CA, Kaplan JL, de Garavilla L and Dalsey WC. Use of tube condensation as an indicator of endotracheal tube placement. Ann Emerg Med. 1998; 31:575-8. | Article | PubMed

8. Clyburn $\mathrm{P}$ and Rosen M. Accidental oesophageal intubation. $\mathrm{Br} \mathrm{J}$ Anaesth. 1994; 73:55-63. | Article | PubMed

9. Li J. Capnography alone is imperfect for endotracheal tube placement confirmation during emergency intubation. J Emerg Med. 2001; 20:2239. | Article | PubMed

10. Salem MR and Baraka AS. Confirmation of tracheal intubation. In: Hagberg CA, ed. Benumof and Hagberg's Airway Management. 3rd ed. Philadelphia: Elsevier Saunders, 2013; 657-684.

11. Sakles JC, Laurin EG, Rantapaa AA and Panacek EA. Airway management in the emergency department: a one-year study of 610 tracheal intubations. Ann Emerg Med. 1998; 31:325-32. | Article | PubMed

12. Schwartz DE, Matthay MA and Cohen NH. Death and other complications of emergency airway management in critically ill adults. A prospective investigation of $\mathbf{2 9 7}$ tracheal intubations. Anesthesiology. 1995; 82:367-76. | Article | PubMed

13. Knapp S, Kofler J, Stoiser B, Thalhammer F, Burgmann H, Posch M, Hofbauer R, Stanzel M and Frass M. The assessment of four different methods to verify tracheal tube placement in the critical care setting. Anesth Analg. 1999; 88:766-70. | Article | PubMed

14. Grmec $S$. Comparison of three different methods to confirm tracheal tube placement in emergency intubation. Intensive Care Med. 2002; 28:701-4. | Article | PubMed

15. Hsu CC, Lee WJ, Wu YL, Guo HR and Lin HJ. Assessment of a new method to distinguish esophageal from tracheal intubation by measuring the endotracheal cuff pressure in a porcine model. Acad Emerg Med. 2005; 12:1153-7. | Article | PubMed

16. Lin HJ, Chen KT, Foo NP, Hsu CC and Guo HR. Detection of oesophageal intubations using cuff pressures in a pig trachea oesophagus model. $\mathrm{Br} J$ Anaesth. 2007; 99:740-3. | Article | PubMed

17. Russo M, Schaner D and Osborn I. Comparison of tracheal and esophageal compliance curves in human cadaveric subjects. Semin Cardiothorac Vasc Anesth. 2010; 14:60-1. | Article I PubMed

18. Kamel KS, Lau $G$ and Stringer MD. In vivo and in vitro morphometry of the human trachea. Clin Anat. 2009; 22:571-9. | Article I PubMed

19. Schaller RJ, Huff JS and Zahn A. Comparison of a colorimetric endtidal $\mathrm{CO} 2$ detector and an esophageal aspiration device for verifying endotracheal tube placement in the prehospital setting: a six-month experience. Prehosp Disaster Med. 1997; 12:57-63. I Article I PubMed

20. Breatnach $E$, Abbott $G C$ and Fraser RG. Dimensions of the normal human trachea. AJR Am J Roentgenol. 1984; 142:903-6. | Article | PubMed

21. Jaensson M, Olowsson LL and Nilsson U. Endotracheal tube size and sore throat following surgery: a randomized-controlled study. Acta Anaesthesiol Scand. 2010; 54:147-53. | Article | PubMed

22. Higgins PP, Chung F and Mezei G. Postoperative sore throat after ambulatory surgery. Br J Anaesth. 2002; 88:582-4. | Article | PubMed

\section{Citation:}

Goentzel BJ, Larsen E, Shelburne N, Tolbert D, Culp Jr WC and Johnston WE. Distinguishing esophageal from endotracheal intubation by measuring endotracheal tube cuff pressure. Emerg Med Health Care. 2013; 1:3. http://dx.doi.org/10.7243/2052-6229-1-3 